\title{
LA RENOVACIÓN DEL CONSERVADURISMO TRADICIONAL A TRAVÉS DE NUEVOS PARTIDOS. EL CASO DEL CENTRO DEMOCRÁTICO EN COLOMBIA, 2014-2018*
}

The renewal of traditional conservatism through new parties. The case of Centro Democrático in Colombia, 2014-2018

\author{
Juan Carlos Rodríguez-Raga \\ Departamento de Ciencia Política \\ Universidad de los Andes \\ juanrodr@uniandes.edu.co \\ https://orcid.org/0000-0002-6491-478X \\ Laura Wills-Otero \\ Departamento de Ciencia Política \\ Universidad de los Andes \\ 1.wills21@uniandes.edu.co \\ https://orcid.org/0000-0001-6330-8975
}

Recibido: 4/2/2021

Aceptado: 14/5/2021

\footnotetext{
* Agradecemos a Juan Guillermo Cabal, Santiago Chavarriaga, David Córdoba y Wilson Forero, estudiantes del pregrado de Ciencia Política de la Universidad de los Andes, por su valiosa asistencia en esta investigación. Juan Carlos Rodríguez-Raga agradece, además, a la Facultad de Ciencias Sociales de esta Universidad por la obtención de un STAI (semestre de trabajo académico independiente) durante la primera mitad de 2020, que permitió completar este artículo. Por otra parte, queremos agradecer los comentarios de quienes participaron en el Coloquio del Departamento de Ciencia Política de la Universidad de los Andes (21 de agosto de 2020) donde presentamos una versión preliminar de este manuscrito, así como las muy valiosas sugerencias de dos árbitros anónimos que lo revisaron para la Revista Uruguaya de Ciencia Política. Gracias a estos comentarios y sugerencias este artículo es considerablemente mejor que en sus versiones anteriores.
} 
Resumen: Desde inicios de la década de 1990, un porcentaje importante de partidos políticos tradicionales en América Latina ha decaído en su poder político y electoral (Wills-Otero, 2016). Los partidos conservadores de diferentes países sufrieron un proceso de desapego por parte de su electorado, tras reformas neoliberales que, en muchos casos, desmejoraron las condiciones de vida de los ciudadanos. Votantes y partidos se desconectaron. Colombia no fue ajena a ese problema. Los partidos tradicionales decayeron electoralmente desde la promulgación de la carta política aperturista de 1991. En años recientes, un nuevo partido de ideología de derecha, el Centro Democrático (CD), bajo la dirección del expresidente Álvaro Uribe, ha logrado conquistar a importantes sectores del electorado. ¿Cuáles han sido los factores determinantes de este éxito electoral reciente? Nuestro análisis de los datos a nivel municipal de las dos últimas elecciones nacionales sugiere que el $\mathrm{CD}$ ha sabido atraer de manera distintiva a los votantes de los municipios del país donde el tradicional Partido Conservador ejerció su dominio durante gran parte del siglo veinte.

Palabras clave: derecha, Centro Democrático, Colombia, partidos tradicionales, identidades políticas

Abstract: Since the early 1990s, a significant percentage of traditional political parties in Latin America have declined in political and electoral power (Wills-Otero, 2016). Conservative parties in different countries suffered a process of disaffection on the part of their electorate, following neoliberal reforms that, in many cases, worsened the living conditions of citizens. Voters and parties became disconnected. Colombia was no stranger to this problem. Traditional parties have declined electorally since the enactment of the 1991 constitution that opened the political arena. In recent years, a new right-wing party, the Centro Democrático (CD), under the leadership of former President Álvaro Uribe, has managed to win over important sectors of the electorate. What have been the determinants in this recent electoral success? Our analysis of municipal-level data from the last two national elections suggests that the $\mathrm{CD}$ has been able to appeal in a distinctive way to voters in the nation's municipalities where the traditional Conservative Party held sway for much of the twentieth century.

Keywords: right-wing, Centro Democrático, Colombia, tradicional political parties, political identities 


\section{Introducción}

En América Latina, diferentes países han tenido a lo largo del tiempo partidos políticos conservadores, conceptualizados como aquellos que extraen su base electoral de los estratos sociales y económicos más altos de la sociedad (Gibson, 1996); como partidos que orientan sus acciones a partir de la idea del rol disminuido del Estado (Luna y Rovira-Kaltwasser 2014), o como aquellos que se ubican en el centro-derecha del espectro ideológico y que tienen dos características, a saber: económicamente, promueven los derechos de propiedad sobre la redistribución y el libre mercado sobre el estatismo; y socialmente, promueven el status-quo de las normas e instituciones existentes (Loxton, 2021). Muchos de estos partidos movilizan apoyo electoral multiclasista, lo que hace que sus políticas no siempre coincidan con sus principios fundacionales. Sus trayectorias, niveles de injerencia en la política, y agendas programáticas han sido variadas (Middlebrook, 2000).

En la región existen partidos conservadores que han perdurado varias décadas (más de un siglo y medio en el caso de Colombia) y hay otros de más reciente formación (v.g., Argentina). En algunos países, la influencia y el poder de estos partidos ha sido significativa en diferentes momentos (ejemplos: Chile, Colombia), mientras que, en otros, su poder tanto electoral como político no ha sido muy significativo (v.g., Brasil, Perú, y tradicionalmente Argentina) (Middlebrook, 2000). En la década de 1980 emergieron nuevos partidos de derecha en los procesos de transición hacia regímenes democráticos. Sin embargo, solamente en pocos países (ejemplo: Chile y El Salvador) pudieron consolidarse (Loxton, 2021). Durante la década de 1990, partidos conservadores tradicionales y nuevos sufrieron un declive en su poder electoral tras la introducción de reformas de libre mercado que produjeron altos niveles de desigualdad y pobreza (Roberts, 2012). En la mayor parte de la región, esta coyuntura crítica de reformas neoliberales desalineó a los sistemas partidistas al generar incertidumbre sobre los compromisos de los partidos. Esta incertidumbre erosionó los vínculos programáticos entre los partidos y los votantes, y generó movimientos antisistema, formas de protesta política y social, y el surgimiento de outsiders políticos (Luna, 2014). El desalineamiento produjo gran volatilidad electoral, y muchos votos que antes recibían los partidos tradicionales fueron atraídos por nuevos partidos y movimientos políticos (Roberts, 2012).

En Colombia, el Partido Conservador (PC), uno de los partidos más antiguos de la región (surgió en 1849), se repartió, hasta inicios de los años 1990, la casi totalidad del poder político y electoral con el otro partido tradicional, el Partido Liberal (PL), creado en 1848. A partir de 1991, como consecuencia de la expedición 
de una nueva constitución política que promovía la apertura democrática, el hasta entonces sistema bipartidista se transformó en uno multipartidista. Los partidos tradicionales empezaron a perder poder político y electoral (Wills-Otero, 2015). El último año en el que alguno de ellos —el PC— ganó las elecciones presidenciales fue en 1998. En la primera década del nuevo milenio (2000), estos partidos perdieron la mayoría del poder que habían acumulado durante más de un siglo en el Congreso de la República. El PC pasó a ser un partido minoritario que, desde 2002, decidió integrar de manera estratégica, e independientemente del presidente de turno, las coaliciones del gobierno.

En este escenario, en 2002, Álvaro Uribe Vélez fue elegido presidente del país a través de su movimiento Primero Colombia. Su agenda programática estuvo enfocada primordialmente en combatir militarmente a los grupos guerrilleros existentes, a través del fortalecimiento de las fuerzas militares. A esta iniciativa la llamó «Seguridad Democrática» y la complementó con otras políticas que estuvieron alineadas con la derecha del espectro ideológico (Wills-Otero, 2014). Los otros pilares en los que se sustentó su agenda programática fueron los siguientes: la confianza inversionista; la cohesión social; un Estado austero y descentralizado; y el diálogo popular.

En 2006, tras una reforma constitucional que permitió la reelección presidencial, Uribe Vélez volvió a ganar las elecciones a través del Partido de Unidad Nacional (Partido de la U). Durante el segundo cuatrienio continuó con su programa de gobierno, mediante el que logró debilitar significativamente a los grupos guerrilleros, en particular a las Fuerzas Armadas Revolucionarias de Colombia-EP (FARC-EP). Tras un cuatrienio fuera del poder electoral, en 2014, aspiró a un escaño en el Senado de la República a través de otro partido que fue creado, en 2013, bajo su liderazgo: el Centro Democrático (CD). Este partido participó por primera vez en las elecciones nacionales de 2014 y posteriormente en las regionales de 2015. En los comicios presidenciales obtuvo cerca de siete millones de votos que representaron el 44,98\% del total de la votación, mientras que en las elecciones al Senado de la República se constituyó en la segunda fuerza, con poco más de dos millones de votos, equivalentes al 14,3\% de la representación. En la Cámara de Representantes, el partido logró el 9,5\% de los escaños. En los comicios regionales de 2015 y locales logró elegir algunos gobernadores, cincuenta alcaldes, y varios escaños en asambleas departamentales y concejos municipales. En 2018 y 2019 volvió a participar en elecciones nacionales y subnacionales, respectivamente. En las primeras, ganó la presidencia en la segunda vuelta electoral con su candidato Iván Duque, quien obtuvo la votación más alta en la historia del país con más de diez millones de votos (54\%). En las elecciones legislativas, el partido aumentó la votación tanto en Senado como en Cámara, con respecto a las que 
había obtenido cuatro años antes, y se constituyó en la primera y segunda fuerza política, respectivamente, con alrededor del $16 \%$ de la representación. Finalmente, en los comicios regionales y locales de 2019, el CD también obtuvo escaños en los diferentes cargos; no obstante, su éxito no fue tan contundente como en las elecciones nacionales. Estos datos evidencian el éxito y crecimiento que el $\mathrm{CD}$ ha tenido en las elecciones en las que ha participado desde su creación. El liderazgo y postura ideológica de derecha de Uribe Vélez han influido de forma importante en este desempeño, al atraer a votantes nuevos y viejos que coinciden con su proyecto político (García Sánchez y Rodríguez-Raga, 2019; Ortiz-Ayala y García-Sánchez, 2014).

En este escenario, la pregunta de investigación que nos interesa responder en este artículo es la siguiente: ¿cuáles han sido los factores determinantes del caudal electoral del $\mathrm{CD}$ en las elecciones nacionales en las que ha participado desde su creación en 2013? Nuestro argumento central sugiere que el CD ha logrado atraer el apoyo de ciudadanos que habitan en lugares donde en el pasado el PC tenía mayorías electorales. Acá planteamos que la creación del CD, con una explícita agenda conservadora en el plano político, económico y social, y con un líder tan popular como Uribe Vélez, se convirtió en una alternativa atractiva para algunos simpatizantes del desdibujado y desideologizado PC que dejaron de sentirse identificados con este.

Ofrecemos evidencia empírica de la forma como el $\mathrm{CD}$ ha logrado atraer a porciones importantes del electorado en lugares que, durante gran parte del siglo veinte, constituyeron la base del conservatismo en Colombia. Este estudio de caso puede dar luces para analizar otros países de América Latina en los que partidos conservadores tradicionales se han debilitado como consecuencia de su desconexión con su electorado. ¿Existen alternativas para esos votantes que representen sus intereses políticos y respondan a sus demandas?

Para responder la pregunta formulada más arriba, analizamos los resultados de las elecciones presidenciales y legislativas de 2014 y 2018. Los datos los observamos desagregados en el nivel municipal, puesto que nos interesa analizar variables contextuales, estructurales y políticas, y ver el impacto que tienen sobre el desempeño electoral de este partido en esas entidades territoriales. ${ }^{1}$

El artículo está organizado de la siguiente manera. En la siguiente sección hacemos una revisión de la literatura de política comparada sobre realineamiento electoral, adaptación de los sistemas de partidos y formación de partidos, espe-

1 Colombia está dividida política y administrativamente en 33 departamentos — más el Distrito Capital de Bogotá. Los departamentos, a su vez, están divididos en municipios (de los que hay, en el país, un total de 1204). 
84 | La renovación del conservadurismo tradicional a través de nuevos partidos.

cialmente aquellos con ideología de derecha. En una tercera sección, reseñamos brevemente los estudios que existen en torno al desempeño electoral del Centro Democrático, y planteamos nuestro argumento teórico con mayor detalle. La cuarta sección describe la estrategia metodológica y los datos de los que hacemos uso para responder a la pregunta de investigación. En la quinta sección presentamos los resultados de los análisis y las estimaciones. La sección final ofrece conclusiones y plantea algunas ideas sobre cómo este estudio puede ser útil para abordar otros casos de partidos conservadores latinoamericanos que intentan resurgir tras sus declives, así como de las identidades políticas asociadas a estos.

\section{Teorías de realineamiento electoral}

En América Latina, los partidos políticos han sido fundamentales en el desarrollo de los regímenes democráticos. A través de ellos se han organizado la mayoría de las elecciones nacionales y subnacionales que han tenido lugar en la tercera ola de democratización. En años recientes, el interés por comprender el desempeño político y la complejidad organizativa de estos actores ha sido creciente en América Latina. Algunos académicos se han interesado por comprender por qué algunos partidos tradicionales han perdurado, mientras que otros han colapsado. En estos estudios, la estructura de las organizaciones internas, sus niveles de democracia interna, las diferentes funciones que cumplen, y la identidad partidista, son variables que explican la variación en los resultados, en contextos de cambios estructurales y políticos profundos (Cyr, 2016; Lupu, 2016, Wills-Otero, 2015, 2020). Partidos más jerárquicos, menos democráticos en sus procesos de selección de candidatos, ineficientes en cumplir al menos una de sus varias funciones, y con vínculos débiles entre sus miembros y sus votantes, son los que más altas probabilidades tienen de colapsar o de volverse insiginificativos. En cambio, partidos con estructuras internas más horizontales, más democráticos, capaces de responder una o más de sus funciones, y con fuertes vínculos con su electorado, tienen más probabilidades de sobrevivir. Otros estudios se han centrado en comprender los factores que explican la vitalidad de los partidos (Rosenblatt, 2018), y cómo en ese resultado las funciones de coordinación horizontal y agregación vertical de intereses son fundamentales (Luna et al., 2020).

Otros trabajos se han enfocado en entender los esfuerzos frecuentes para crear nuevos partidos, y las altas posibilidades que existen para que estos fracasen, o para que no se conviertan en actores con un peso político y electoral significativo en el largo plazo. Levitsky et al. (2016), plantean que los partidos nuevos que han sido exitosos en la región, han emergido después de períodos de conflictos extraordinarios, los cuales incluyen luchas revolucionarias, guerras civiles, 
movilizaciones populares de larga escala, y represión autoritaria. Tales conflictos contribuyen a la formación exitosa de partidos de diferentes maneras. En primer lugar, fortalecen los vínculos partidarios (Lupu, 2016; Rosenblatt, 2018). En segundo lugar, promueven la construcción de organizaciones con el apoyo de bases sólidas y activas (Samuels, 2006; Samuels y Zucco, 2014). Finalmente, promueven la cohesión interna (Levitsky y Way, 2012). Por otro lado, este resultado es más probable cuando sus fundadores heredan la marca o la infraestructura organizacional de movimientos sociales (Anria, 2019), de exguerrillas, o de anteriores dictaduras (Levitsky et al., 2016).

Entre los partidos políticos nuevos que han sido exitosos, se incluyen partidos conservadores de la nueva derecha latinoamericana que, además, se caracterizan por tener vínculos con los regímenes autoritarios existentes antes de 1978. Estos partidos son la Unión Democrática Independiente (UDI) y Renovación Nacional (RN) en Chile, la Alianza Repúblicana Nacionalista (ARENA) en El Salvador, y el Partido del Frente Liberal (PFL) en Brasil (Loxton, 2016). Su éxito, según Loxton (2016, 2021), se debe a la herencia autoritaria que los proveyó de diferentes recursos, tales como una marca, una organización territorial, fuentes de cohesión interna, redes clientelares, y fuentes de financiación. Otros partidos conservadores de derecha, desligados de gobiernos autoritarios, que surgieron en este período y que han ganado elecciones, son Propuesta Republicana (PRO) en Argentina (Vommaro, 2019) y Centro Democrático (CD) en Colombia. El primero surgió en el contexto de la crisis económica y social por la que atravesó Argentina en 2001 y 2002. Dicha crisis generó una oportunidad política para que actores identificados con la centro-derecha constituyeran su propia fuerza, alejada de los desprestigiados partidos tradicionales (Vommaro, Morresi y Belloti, 2015). Así, surgieron los partidos Recrear y PRO. Este último nació con una agenda programática amplia, que incluyó las ideas promercado de centro-derecha, y acogió cuadros provenientes de ONG profesionalizadas, y fundaciones; de empresarios y cuadros de alta gerencia del mundo privado, e incluso de los desprestigiados partidos políticos (Vommaro, 2019, p. 99). La ventana de oportunidad que proporcionó la crisis se combinó con la «polarización política que se dio en los últimos años del peronismo kirchnerista y que activó un 'pánico moral' (Cohen, 1972) respecto de una posible deriva 'chavista' de Argentina» (Vommaro, 2019, p. 109). Esta opción partidista que surgió localmente en la ciudad de Buenos Aires, logró unificar la centro-derecha, obtener cobertura nacional, y ganar las elecciones presidenciales de 2015 con su candidato y fundador Mauricio Macri (Vommaro, 2017). 
86 | La renovación del conservadurismo tradicional a través de nuevos partidos.

\section{El desempeño electoral del Centro Democrático}

Los trabajos mencionados anteriormente son útiles para reflexionar en torno al surgimiento del CD en Colombia. Este partido emergió en 2013, bajo el liderazgo del expresidente Uribe Vélez en una coyuntura de intensa polarización política, que se dio como resultado de las negociaciones de paz entre el gobierno de Juan Manuel Santos y las Fuerzas Armadas Revolucionarias de Colombia (FARC-EP) en la Habana (Cuba), a las que Uribe Vélez se opuso desde su inicio. Ante la posibilidad de que Santos aspirara y lograra la reelección en 2014 y, con ella, pudiera continuar y eventualmente culminar las negociaciones de paz, la creación de un nuevo partido que desafiara tales propósitos se convirtió en la ambición inmediata de Uribe Vélez y sus seguidores. El éxito electoral que había acumulado el expresidente en los dos comicios en los que compitió y ganó con amplia ventaja (2002 y 2006); la inusitadamente alta popularidad que mantuvo a lo largo de los dos cuatrienios de su mandato (García-Sánchez y Rodríguez-Raga, 2019); y el apoyo que recibió por parte de personalidades con alto perfil en la política del país, se constituyeron en factores decisivos para formar un partido cuyo propósito inmediato sería el de participar en elecciones nacionales para impedir, en el mejor de los casos, la reelección de Santos y la continuidad del proceso de paz o, en un escenario menos optimista, para desafiar al presidente desde la oposición con Uribe Vélez y sus copartidarios en ambas cámaras del Congreso de la República. Como en Argentina con el PRo, los cofundadores y militantes del nuevo partido, provenían de los partidos tradicionales —especialmente del $\mathrm{PC}^{2}$ - y de otros de más reciente formación, de sectores empresariales, y de un electorado heterogéneo. De manera similar, el pánico moral por el castrochavismo o la instalación del socialismo del siglo veintiuno en el país, le dio fuerza al nuevo partido.

La organización del partido es jerárquica, personalista y caudillista (Atehortúa, 2016; Orjuela, 2015). Su líder orientador es el expresidente Uribe Vélez, y es él quien determina la agenda programática de la organización, quien impone disciplina interna en el Congreso (Cifuentes y Pino, 2018; Congreso Visible, 2020, Gamboa, 2019), y quien orienta a los votantes en los procesos electorales. Uno de los pilares fundamentales que ha orientado el accionar del partido, tanto en la arena política como en la electoral, ha sido la Seguridad Democrática, entendida ante todo como una «lucha frontal contra los alzados en armas [y también como] combate a cualquier forma de criminalidad» (Losada y Liendo, 2016, p. 50).

2 Políticos y asesores del CD entrevistados para este y otro trabajo, afirmaron que varios de los integrantes del nuevo partido, así como de sus votantes, provienen del PC. 
El desempeño electoral del partido ha recibido la atención de algunos académicos. Algunos trabajos han sido de carácter descriptivo, y se han concentrado en analizar, por ejemplo, la geografía de los votos que obtiene el CD. Hay regiones del país en las que ha sido más exitoso (Bogotá y Antioquia), y hay zonas donde su desempeño es más modesto e incluso algunas en las que se ha abstenido de participar. Losada y Liendo (2016) concluyen que las circunscripciones en las que más votos obtuvo en las elecciones legislativas de 2014 fueron las más pobladas o de magnitud de distrito más grande, mientras que no presentó candidatos en las regiones escasamente pobladas y con influencia activa de las FARC.

En una vena más analítica, en un estudio que indagó por los efectos de los contextos violentos sobre el voto (Weintraub, Vargas y Flores, 2015) se encontró que Oscar Iván Zuluaga, candidato presidencial del CD en 2014, tuvo un mejor desempeño en los municipios con menores y con mayores niveles de violencia insurgente, y le fue menos bien en los municipios con moderados niveles de violencia. Estos últimos, según los autores, fueron los que conquistó el entonces presidente Juan Manuel Santos.

Algunos autores han encontrado correlaciones entre las condiciones estructurales y contextuales de los municipios y el desempeño del CD. Losada y Liendo (2016) encuentran que este partido obtuvo considerablemente más votos en departamentos y municipios con menos necesidades básicas insatisfechas (NBI) y con un mayor nivel de riqueza (medida mediante el PIB per cápita). Otros trabajos han resaltado que la clara ideología de derecha y conservadora del nuevo partido explica, al menos parcialmente, sus resultados electorales (Wills-Otero, 2014).

La crisis de representación democrática padecida por el sistema de partidos desde la década de 1990, como consecuencia de la clientelización y desideologización de las organizaciones políticas (Mainwaring, Bejarano y Pizarro, 2008), podría verse mitigada con el surgimiento de nuevos partidos con claras ideologías y agendas programáticas que tuvieran posibilidades de ganar elecciones y de influir en los procesos políticos (Orjuela, 2015). En un contexto en el que las organizaciones políticas existentes sufren de desprestigio por su incapacidad para coordinarse horizontalmente y para agregar intereses de forma vertical - ocasionado esto por fuertes personalismos- (Luna et. al., 2020), la consolidación de opciones que sean capaces tanto de identificar demandas ciudadanas como de traducirlas en políticas consistentes con dichos intereses se presenta como una ventana de oportunidad para quienes lideran dichos procesos.

El contexto político polarizado, originado por la ruptura entre Uribe Vélez y Santos en 2012, y exacerbado como consecuencia del Plebiscito por la Paz del mes de octubre de 2016, determinó que la mayoría de los partidos políticos 
tomaran postura a favor o en contra del proceso de paz y, al hacerlo, que fueran asociados por la ciudadanía como organizaciones con ideologías políticas discernibles. Los que defendían las negociaciones de paz entre el Gobierno nacional y las FARC se asociaban más con el lado izquierdo del espectro ideológico, mientras que los que estaban en contra y defendían su derrota militar se identificaban con la derecha. El CD, a pesar de auto definirse como una opción de centro — se llamó inicialmente Puro Centro Democrático-, se concibió como la organización más derechizada del sistema. Y aunque el PC apoyó a Santos en su coalición durante los dos mandatos, el fraccionamiento del partido se hizo evidente cuando varios de sus líderes apoyaron a Uribe Vélez durante el proceso de formación del CD (Losada y Liendo, 2016) y, más adelante, cuando el candidato presidencial Iván Duque invitó a Martha Lucía Ramírez — conservadora y excandidata en 2014 a ser su fórmula vicepresidencial en la campaña de $2018 .^{3}$ Desde el triunfo de Duque, el PC decidió acompañar al CD en la coalición del gobierno en el Congreso de la República. Por su parte, el presidente electo asignó cuotas burocráticas al partido en el gabinete ministerial.

Los dos partidos se han mostrado cercanos en sus agendas políticas, pero el $\mathrm{CD}$ ha logrado representar las tendencias conservadoras del país de forma más efectiva que el PC. Su organización jerárquica ha mostrado coherencia interna entre sus miembros. Por su parte, aunque también tiene una organización jerárquica (Wills-Otero, 2015), el PC está fraccionado internamente. No hay coherencia interna entre sus miembros, y los intereses que muchos de ellos promueven son más de carácter clientelar que programático (Wills-Otero, Ortega y Sarmiento, 2021). A pesar de esto, también es cierto que hay militantes y líderes que defienden ideales conservadores en sus programas de gobierno. Sin embargo, la fragmentación interna, y la incapacidad de sus líderes para coordinar a sus políticos durante procesos electorales y entre ellos, y de agregar intereses de un electorado difuso, dificulta la posibilidad de plasmar dichos ideales en políticas públicas concretas.

El debilitamiento del PC se ha visto reflejado en su pérdida de poder electoral, sobre todo desde la adopción de la Constitución política de 1991, cuando la apertura política transformó el sistema bipartidista en uno multipartidista. Hasta ese momento y desde su creación a mediados del siglo xIx, este partido fue uno de los dos más poderosos del sistema político junto a su principal competidor, el PL. Desde su creación hasta finales de los 1950, estas dos organizaciones se

3 En las elecciones legislativas de 2018 se llevaron a cabo dos consultas internas para elegir a los candidatos presidenciales de la derecha y de la izquierda. En la primera, «La Gran Consulta por Colombia», participaron Iván Duque por el Centro Democrático y Martha Lucía Ramírez y Alejandro Ordoñez por el Partido Conservador. El primero obtuvo el $67,8 \%$, frente al $25,7 \%$ y $6,4 \%$ respectivamente, y fue electo como el candidato del $\mathrm{CD}$. 
enfrentaron de manera intermitente en guerras civiles y conflictos violentos. Dos subculturas políticas, que se diferenciaban en sus ideales y agendas programáticas, y que se enraizaban en diferentes regiones, determinaron el destino político del país (González,1997; Melo, 2018). Colombia era un país dividido entre liberales rojos y conservadores azules que controlaron desde inicios de los años 1930 el poder político en determinados municipios y regiones (Pinzón de Lewin, 1988). Los presupuestos ideológicos de estos partidos empezaron a desdibujarse al iniciarse el Frente Nacional. La repartición equitativa de la burocracia estatal entre los dos, y la rotación asegurada del poder político durante dieciséis años, desincentivó a estas organizaciones a competir por programas de gobierno diferenciados. Fue durante esos años que la clientelización y desideologización de uno y otro se profundizó más (Hartlyn, 1993; Archer, 1995). A pesar de lo anterior, el arraigo de los partidos en una porción significativa de municipios del país le permitió a uno y otro mantener una porción de su electorado estable, y sobrevivir hasta la fecha. Como se dijo antes, la polarización profunda que vivió el país a raíz del proceso de paz entre el gobierno de Santos y las FARC de alguna forma revivió la ideologización de los partidos —al menos de algunos-, así como de los votantes. Una porción significativa del electorado del PC encontró afinidades con el claro conservadurismo de derecha del CD, se volvió atractivo para una porción significativa del electorado del PC que desde hacía años percibía deslegitimizada a esta organización.

Por todo lo anterior, el argumento central que planteamos en este artículo es que, más allá de las condiciones estructurales de pobreza o afluencia, y de las afectaciones por el conflicto armado, una porción significativa de los enclaves electorales que mantuvo el PC durante gran parte del siglo veinte encontró en el claro conservadurismo de derecha del CD una expresión política atractiva. Aunque algunos estudios han analizado la geografía del voto por el CD (Milanese et.al. 2016), hasta el momento no hay trabajos que hayan probado empíricamente el argumento que proponemos. Así, la hipótesis principal que acá planteamos es la siguiente:

H1: El caudal de votantes por el CD es mayor en los municipios que tradicionalmente estuvieron controlados electoralmente por el Partido Conservador en relación con los municipios con otras tradiciones partidistas.

Además de la expectativa expresada en la hipótesis anterior, de la literatura sobre el CD reseñada arriba se derivan las dos siguientes hipótesis alternativas:

$\mathrm{H} 2$ : El CD recibe un mayor apoyo electoral en los municipios comparativamente menos afectados por el conflicto armado.

H3: Los resultados del CD son mejores en los municipios más desarrollados $\mathrm{y}$ con menores niveles de pobreza. 


\section{Estrategia empírica}

El argumento central de este artículo es que - más allá de lo que han encontrado previamente otros autores sobre los lugares donde este partido tiene apoyo electoral - el $\mathrm{CD}$ ha obtenido comparativamente mejores resultados en las elecciones en las que ha competido en aquellos municipios que fueron fortines conservadores durante gran parte del siglo xx, que en municipios con otras tradiciones políticas. Para evaluar empíricamente este argumento y contrastarlo con las hipótesis que relacionan el desempeño electoral del CD con el conflicto o con condiciones estructurales de contexto, construimos una base de datos a nivel municipal a partir de múltiples fuentes.

Por una parte, para medir el fenómeno que nos interesa en este artículo - nuestra variable dependiente-, hacemos uso de los datos electorales provistos por la Registraduría Nacional del Estado Civil para los comicios en los que ha participado como partido el CD, es decir, las elecciones de Congreso de 2014 (9 de marzo) y 2018 (11 de marzo) y las elecciones presidenciales de 2014 ( 25 de mayo) y 2018 (27 de mayo). Para el análisis de cada una de estas elecciones, la variable de interés es el porcentaje de votos obtenido por el $\mathrm{CD}$ en cada municipio. ${ }^{4}$

La tabla 1 muestra los estadísticos descriptivos de estas variables. ${ }^{5}$ Estos datos evidencian el avance del CD entre las elecciones de 2014 y las de 2018. Excepto en el caso del Senado de la República (cuyo promedio descendió levemente entre 2014 y 2018 , de $14,4 \%$ a 13,5\%), los porcentajes de votos obtenidos en los municipios aumentaron en promedio en los cuatro años transcurridos entre estos dos momentos electorales.

4 En la medida en que el objetivo de este artículo es examinar el capital electoral propio del CD y los factores asociados con este, solo tenemos en cuenta la primera vuelta de la elección presidencial, dado que en general un balotaje involucra decisiones de voto, tanto a favor de un candidato como en contra de otro. En otras palabras, los votos obtenidos por los candidatos del CD en una segunda vuelta no son, necesariamente, todos atribuibles al capital electoral de ese partido.

5 En esta tabla y en los análisis que siguen, los porcentajes están calculados como fracciones, de 0 a 1. 
Tabla 1. Resultados del CD en las elecciones 2014 y 2018. Estadísticos descriptivos

\begin{tabular}{lccccc}
\hline $\begin{array}{c}\text { Elección } \\
(\% \text { del } \mathbf{~ C D})\end{array}$ & $\mathbf{N}$ & Media & $\begin{array}{c}\text { Desv. } \\
\text { Est. }\end{array}$ & Mín. & Máx. \\
\hline $1 .{ }^{\text {a }}$ vuelta presidencial 2014 & 1,122 & 0,347 & 0,168 & 0,000 & 0,825 \\
\hline $1 .{ }^{\text {a vuelta presidencial 2018 }}$ & 1,120 & 0,478 & 0,187 & 0,019 & 0,883 \\
\hline Senado 2014 & 1,119 & 0,144 & 0,119 & 0,001 & 0,772 \\
\hline Senado 2018 & 1,119 & 0,135 & 0,113 & 0,002 & 0,764 \\
\hline Cámara 2014 & 999 & 0,085 & 0,080 & 0,000 & 0,571 \\
\hline Cámara 2018 & 1,080 & 0,138 & 0,118 & 0,001 & 0,794 \\
\hline
\end{tabular}

Fuente: Registraduría Nacional del Estado Civil; cálculo de los autores.

La tabla 2, a su vez, muestra que el apoyo electoral del CD en los municipios colombianos ha sido relativamente consistente entre elecciones y a lo largo del tiempo. Existe, por ejemplo, una correlación de 0,85 entre el porcentaje de votos municipal, y de 0,79 entre las dos elecciones al Senado. Igualmente, existe una correlación de 0,8 en los resultados obtenidos por el CD en las elecciones las dos cámaras del Congreso en $2018 .{ }^{6}$

Tabla 2. Correlaciones de los resultados electorales del CD entre las diferentes elecciones (2014-2019)

\begin{tabular}{lcccccc}
\hline $\begin{array}{c}\text { Elecciones } \\
\text { (\% de votos) }\end{array}$ & $\mathbf{( 1 )}$ & $\mathbf{( 2 )}$ & $\mathbf{( 3 )}$ & $\mathbf{( 4 )}$ & $\mathbf{( 5 )}$ & $\mathbf{( 6 )}$ \\
\hline (1) Pres. 2014 & 1,000 & & & & & \\
\hline (2) Pres. 2018 & $0,848^{*}$ & 1,000 & & & & \\
\hline (3) Sen. 2014 & $0,705^{*}$ & $0,594^{*}$ & 1,000 & & & \\
\hline (4) Sen. 2018 & $0,609^{*}$ & $0,599^{*}$ & $0,787^{*}$ & 1,000 & & \\
\hline (5) Cám. 2014 & $0,474^{*}$ & $0,437^{*}$ & $0,747^{*}$ & $0,611^{*}$ & 1,000 & \\
\hline (6) Cám. 2018 & $0,458^{*}$ & $0,477^{*}$ & $0,624^{*}$ & $0,804^{*}$ & $0,595^{*}$ & 1,000 \\
\hline
\end{tabular}

* significativo al nivel .01

Fuente: Registraduría Nacional del Estado Civil; cálculo de los autores.

6 Todas estas correlaciones entre resultados electorales son significativas al $1 \%$. 
En cuanto a los factores que podrían estar asociados con la variación en el desempeño electoral del $\mathrm{CD}$, por otra parte, nuestro argumento requiere caracterizar de forma sintética la historia político-electoral de los municipios del país. Para esto utilizamos los datos suministrados en Pinzón de Lewin (1989). Este trabajo, a su vez, hace uso de una completa base de datos electorales recopilada por la Universidad de los Andes, que describe el comportamiento político de los municipios colombianos desde los años 1930. La autora examina la historia electoral de cada municipio, para diferentes períodos (antes, durante y después del Frente Nacional), y lo clasifica según si un partido recibió consistentemente una mayoría (simple) en dicho municipio. Con base en este valioso estudio, creamos dos variables que señalan, para cada municipio, si en este hubo mayorías conservadoras o liberales entre 1931 y 1982 . La tabla 3, muestra que, entre 1931 y 1982 , el PC dominó 226 de los actuales 1134 municipios del país (19,9\%), mientras que el PL lo hizo en 428 (37,3\%).

\section{Tabla 3. Distribución del dominio electoral en los municipios de Colombia, 1931-1982}

\begin{tabular}{lcc}
\multicolumn{1}{c}{ Dominio electoral 1931-1982 } & N & \% \\
\hline Mayoría conservadora & 226 & 19,9 \\
Mayoría liberal & 428 & 37,7 \\
Otro/ninguno & 480 & 32,3 \\
\hline
\end{tabular}

Fuente: Pinzón (1989); codificación y elaboración de los autores.

El análisis de la relación simple entre el control conservador durante gran parte del siglo veinte y el desempeño electoral del CD en la segunda década del siglo XXI en los municipios del país brinda preliminarmente respaldo empírico a nuestro argumento $(\mathrm{H} 1)$ de que este partido ha recogido apoyo significativo en aquellos municipios donde el Partido Conservador obtenía mayorías. La tabla 4 muestra el resultado de hacer pruebas $t$ de diferencias de porcentajes promedio de votos del $\mathrm{CD}$ en las diferentes elecciones analizadas entre los municipios, donde el PC tuvo mayorías consistentes entre 1931 y 1982 y aquellos que no. Como se ve en la tabla, en todas las elecciones el porcentaje de votos promedio del CD es mayor en los lugares con tradición conservadora que en los municipios con otras tradiciones políticas. En todos los casos, esta diferencia es significativa (al menos al $10 \%$ ). En algunos casos, esta diferencia es considerable como en la primera vuelta presidencial de 2018 , en la que el CD obtuvo en promedio poco más de 
trece puntos porcentuales más en los municipios tradicionalmente conservadores que en los municipios con otras herencias políticas.

\section{Tabla 4. Diferencias en el porcentaje de votos del CD entre municipios conservadores y municipios no conservadores (pruebas $t$ )}

\begin{tabular}{lcccc}
\hline & $\begin{array}{c}\text { Municipio } \\
\text { sin mayoría } \\
\text { conservadora } \\
(\mathbf{1 9 3 1 - 1 9 8 2 )}\end{array}$ & $\begin{array}{c}\text { Municipio } \\
\text { con mayoría } \\
\text { conservadora } \\
\mathbf{( 1 9 3 1 - 1 9 8 2 )}\end{array}$ & Diferencia & \\
\hline $1 .^{\text {a }}$ vuelta pres. 2014 & 0,326 & 0,431 & 0,105 & $* * *$ \\
\hline $1 .^{\text {a }}$ vuelta pres. 2018 & 0,452 & 0,584 & 0,132 & $* * *$ \\
\hline Senado 2014 & 0,131 & 0,193 & 0,062 & $* * *$ \\
\hline Senado 2018 & 0,124 & 0,176 & 0,052 & $* * *$ \\
\hline Cámara 2014 & 0,075 & 0,120 & 0,045 & $* * *$ \\
\hline Cámara 2018 & 0,129 & 0,173 & 0,044 & $* * *$ \\
\hline
\end{tabular}

Con el fin de evaluar la hipótesis H2, sintetizamos la afectación de los municipios por el conflicto armado identificando aquellos municipios que el acuerdo de paz determinó como receptores de Programas de Desarrollo con Enfoque Territorial (PDET). Estos programas se crearon mediante el decreto 893 de 2017, emitido por el entonces presidente Santos en uso de las facultades extraordinarias para legislar en materias relacionadas con el punto 1 del acuerdo de paz con las FARC. En consecuencia, se crearon estos programas con priorización en 170 municipios de 16 subregiones del país. Estos municipios fueron seleccionados por combinar cuatro características: altos niveles de pobreza, afectación por economías ilícitas (cultivos de uso ilícito y minería ilegal), débil presencia institucional y mayor afectación por el conflicto. Como tales, se trata de municipios donde, en particular, el conflicto fue especialmente crudo.

La H2 sugiere, entonces, que el CD ha sido menos exitoso en captar votantes de estos municipios PDET en comparación con los demás municipios del país. La evidencia sintetizada en la tabla 5 le da un sustento preliminar a esta asociación. En efecto, se ve en la tabla que, para todas las elecciones analizadas, el porcentaje promedio de votos del CD en los 170 municipios PDET fue menor que el promedio en los demás municipios. Esta diferencia es estadísticamente significativa (al 1\%) en todos los casos. De hecho, alcanza poco menos de 16 puntos porcentuales en la primera vuelta presidencial de 2018. 


\section{Tabla 5. Diferencias en el porcentaje de votos del CD entre municipios no PDET y municipios PDET (pruebas $t$ )}

\begin{tabular}{lcccc}
\hline & $\begin{array}{c}\text { Municipio } \\
\text { no PDET }\end{array}$ & $\begin{array}{c}\text { Municipio } \\
\text { PDET }\end{array}$ & Diferencia & \\
\hline $1 .^{\text {a }}$ vuelta pres. 2014 & 0,367 & 0,256 & 0,111 & $* * *$ \\
\hline $1 .{ }^{a}$ vuelta pres. 2018 & 0,507 & 0,349 & 0,159 & $* * *$ \\
\hline Senado 2014 & 0,154 & 0,091 & 0,063 & $* * *$ \\
\hline Senado 2018 & 0,146 & 0,071 & 0,075 & $* * *$ \\
\hline Cámara 2014 & 0,090 & 0,046 & 0,044 & $* * *$ \\
\hline Cámara 2018 & 0,145 & 0,076 & 0,070 & $* * *$ \\
\hline
\end{tabular}

$* * * p<.01 ; * * p<.05 ; * p<.1$

Ahora bien, la tercera hipótesis relaciona las condiciones estructurales y de desarrollo de un municipio con el desempeño electoral del CD en él. Concretamente, la literatura reseñada y recogida en la $\mathrm{H} 3$ establece que este partido obtiene comparativamente mejores resultados en municipios con mayores niveles de desarrollo económico y menores niveles de pobreza.

Con el fin de evaluar esta hipótesis incorporamos a nuestra base los datos del Panel Municipal recopilados a partir de diversas fuentes por el Centro de Estudios sobre Desarrollo Económico (CEDE) de la Facultad de Economía de la Universidad de los Andes. ${ }^{7}$ Del Panel de Características Generales extrajimos el PBI per cápita municipal ${ }^{8}$, como una medida de desarrollo general de cada municipio, el Índice de Pobreza Multidimensional (IPM) total del municipio ${ }^{9}$ y el índice de GINI municipa ${ }^{10}$. Además, del Panel de Agricultura y Tierra usamos el índice de GINI de terrenos ${ }^{11}$ con el fin de examinar la situación de distribución de la tierra en cada municipio. La tabla 6 muestra los estadísticos descriptivos de estas variables.

7 Obtuvimos estos datos consultando la página https://datoscede.uniandes.edu.co/es/catalogo-de-microdata (fecha de consulta: 2 de mayo de 2020).

8 El PIB per cápita municipal está en millones de pesos. Utilizamos los valores de 2009, el último año disponible.

9 Usamos los valores para 2005, el último año disponible.

10 Usamos los valores para 2005, el último año disponible.

11 Usamos los valores para 2012, el último año disponible. 
Tabla 6. Características estructurales de los municipios. Estadísticos descriptivos

\begin{tabular}{lccccc}
\hline $\begin{array}{l}\text { Elección } \\
(\% \text { del } \mathbf{~ C D})\end{array}$ & $\mathbf{N}$ & Media & Desv. Est. & Mín. & Máx. \\
\hline PBI per cápita & 1,097 & 8,19 & 8,82 & 0,19 & 138,08 \\
\hline IPM & 1,098 & 69,14 & 16,26 & 14,27 & 100,00 \\
\hline Gini municipal & 1,043 & 0,454 & 0,034 & 0,394 & 0,568 \\
\hline Gini de terrenos & 962 & 0,691 & 0,107 & 0,293 & 0,998 \\
\hline
\end{tabular}

Fuente: Panel municipal CEDE.

La H3 propone, concretamente, que cuanto más alto sea el PBI per cápita de un municipio, mejor será el desempeño electoral del CD. Por su parte, se espera que, cuanto mayor sea el Índice de Pobreza Multidimensional el partido tendrá menos éxito electoral. Incluimos los índices de desigualdad sin tener una expectativa clara acerca de su efecto sobre los resultados del CD en las elecciones.

\section{Determinantes del desempeño electoral del Centro Democrático}

Con el fin de poner a prueba las hipótesis planteadas, construimos seis modelos de regresión lineal, uno para cada una de las elecciones del orden nacional en las que participó el CD desde su creación en 2013, esto es, dos elecciones presidenciales en primera vuelta (2014 y 2018) y dos elecciones para cada una de las cámaras del Congreso (2014 y 2018). En cada uno de estos modelos, la variable dependiente es el porcentaje de los votos de cada municipio que obtuvo el CD en la elección correspondiente. Como variables independientes incluimos aquéllas que dan cuenta de cada una de las hipótesis planteadas y que describimos en los párrafos anteriores.

Además, incluimos una variable de control que clasifica los municipios según la capacidad estatal con la que cuenta cada uno. Esta variable, que replica la utilizada por el Observatorio de la Democracia en la definición del marco muestral del estudio especial de 2019, se basa en el indicador de desempeño fiscal de cada municipio, construido por el Departamento Nacional de Planeación. La variable de control incluida en nuestros modelos es una dicotómica que toma como punto de corte la mediana de dicho indicador. En otras palabras, con base en su desempeño fiscal, clasificamos la mitad de los municipios como de baja capaci- 
96 | La renovación del conservadurismo tradicional a través de nuevos partidos.

dad estatal (valor 0) y la otra mitad — por encima de la mediana — como de alta capacidad estatal (valor 1). ${ }^{12}$ También controlamos por el tamaño de población de cada municipio, el porcentaje de esta población que vive en zona rural o en centros poblados del municipio ${ }^{13}$, y si el municipio es capital de departamento (incluyendo Bogotá). Los resultados de estos modelos aparecen en la tabla 7.

\section{Tabla 7. Determinantes del desempeño electoral del Centro Democrático, 2014-2018}

\begin{tabular}{|c|c|c|c|c|c|c|}
\hline Variables & $\begin{array}{c}\text { \% Sen. } \\
2014\end{array}$ & $\begin{array}{c}\text { \% Sen. } \\
2018\end{array}$ & $\begin{array}{c}\text { \% Cám. } \\
2014\end{array}$ & $\begin{array}{c}\text { \% Cám. } \\
2018\end{array}$ & $\begin{array}{c}\text { \% Pres. } \\
2014\end{array}$ & $\begin{array}{c}\text { \% Pres. } \\
2018\end{array}$ \\
\hline $\begin{array}{l}\text { Mayorías } \\
\text { conservadoras } \\
(1931-1982)\end{array}$ & $\begin{array}{l}0,0550 * * * \\
(0,00899)\end{array}$ & $\begin{array}{l}0,0339 * * * \\
(0,00800)\end{array}$ & $\begin{array}{l}0,0328 * * * \\
(0,00606)\end{array}$ & $\begin{array}{l}0,0270 * * * \\
(0,00847)\end{array}$ & $\begin{array}{l}0,0875 * * * \\
(0,0140)\end{array}$ & $\begin{array}{c}0,0813 * * * \\
(0,0144)\end{array}$ \\
\hline $\begin{array}{l}\text { Mayorías libe- } \\
\text { rales } \\
(1931-1982)\end{array}$ & $\begin{array}{l}-0,00344 \\
(0,00757)\end{array}$ & $\begin{array}{l}-0,0107 \\
(0,00673)\end{array}$ & $\begin{array}{l}-0,00559 \\
(0,00516)\end{array}$ & $\begin{array}{l}-0,0133^{*} \\
(0,00722)\end{array}$ & $\begin{array}{l}-0,0161 \\
(0,0118)\end{array}$ & $\begin{array}{c}-0,0291 * * \\
(0,0121)\end{array}$ \\
\hline Municipio PDET & $\begin{array}{l}-0,00161 \\
(0,0107)\end{array}$ & $\begin{array}{c}-0,0126 \\
(0,00955)\end{array}$ & $\begin{array}{l}-0,00839 \\
(0,00784)\end{array}$ & $\begin{array}{l}-0,00266 \\
(0,0104)\end{array}$ & $\begin{array}{c}-0,0390 * * \\
(0,0167)\end{array}$ & $\begin{array}{c}-0,0690 * * * \\
(0,0172)\end{array}$ \\
\hline PBI per cápita & $\begin{array}{l}0,000692^{*} \\
(0,000420)\end{array}$ & $\begin{array}{c}0,000923^{* *} \\
(0,000374)\end{array}$ & $\begin{array}{c}0,000624 * * \\
(0,000275)\end{array}$ & $\begin{array}{l}0,00147 * * * \\
(0,000395)\end{array}$ & $\begin{array}{c}0,00202 * * * \\
(0,000656)\end{array}$ & $\begin{array}{l}0,00343 * * * \\
(0,000672)\end{array}$ \\
\hline IPM total & $\begin{array}{c}-0,00268 * * * \\
(0,000285)\end{array}$ & $\begin{array}{c}-0,00206 * * * \\
(0,000253)\end{array}$ & $\begin{array}{c}-0,00140 * * * \\
(0,000190)\end{array}$ & $\begin{array}{c}-0,00191 * * * \\
(0,000269)\end{array}$ & $\begin{array}{c}0,000183 \\
(0,000444)\end{array}$ & $\begin{array}{c}0,000185 \\
(0,000455)\end{array}$ \\
\hline Gini municipal & $\begin{array}{l}-0,136 \\
(0,124)\end{array}$ & $\begin{array}{c}-0,355^{* * *} \\
(0,110)\end{array}$ & $\begin{array}{l}-0,209 * * \\
(0,0992)\end{array}$ & $\begin{array}{c}-0,536^{* * * *} \\
(0,127)\end{array}$ & $\begin{array}{c}-1,116^{* * * *} \\
(0,193)\end{array}$ & $\begin{array}{c}-1,577 * * * \\
(0,198)\end{array}$ \\
\hline Gini de terrenos & $\begin{array}{c}-0,0793 * * \\
(0,0337)\end{array}$ & $\begin{array}{l}-0,0375 \\
(0,0300)\end{array}$ & $\begin{array}{c}-0,0632 * * \\
(0,0246)\end{array}$ & $\begin{array}{c}-0,0673 * * \\
(0,0325)\end{array}$ & $\begin{array}{l}-0,0743 \\
(0,0526)\end{array}$ & $\begin{array}{c}-0,140 * * * \\
(0,0539)\end{array}$ \\
\hline Capacidad statal & $\begin{array}{c}0,00208 \\
(0,00740)\end{array}$ & $\begin{array}{l}-0,00251 \\
(0,00658)\end{array}$ & $\begin{array}{c}0,00173 \\
(0,00502)\end{array}$ & $\begin{array}{c}0,00135 \\
(0,00699)\end{array}$ & $\begin{array}{c}-0,0250 * * \\
(0,0115)\end{array}$ & $\begin{array}{c}-0,0324 * * * \\
(0,0118)\end{array}$ \\
\hline
\end{tabular}

12 Aunque, como se mencionó arriba, la clasificación de un municipio como PDET incluye criterios asociados con la situación económica y la presencia del Estado en dicho municipio, existe una considerable variación entre estos municipios en relación con estas condiciones económicas e institucionales. De hecho, la correlación entre la variable que clasifica los municipios como PDET o no PDET y los indicadores de PBI per cápita, pobreza multidimensional y capacidad estatal no es particularmente alta. Además, los modelos son robustos a la exclusión de la variable PDET; los resultados de dichos modelos sin esta variable son sustantivamente idénticos a los que mostramos en la tabla 7.

13 La población municipal y su discriminación entre casco urbano y zona rural o centro poblado son proyecciones a 2018 hechas por el DANE. (https://www.dane.gov.co/index.php/estadisticas-por-tema/ demografia-y-poblacion/proyecciones-de-poblacion; revisado el 2 de mayo de 2020). 


\begin{tabular}{|c|c|c|c|c|c|c|}
\hline Variables & $\begin{array}{c}\text { \% Sen. } \\
2014\end{array}$ & $\begin{array}{c}\text { \% Sen. } \\
2018\end{array}$ & $\begin{array}{c}\text { \% Cám. } \\
2014\end{array}$ & $\begin{array}{c}\text { \% Cám. } \\
2018\end{array}$ & $\begin{array}{c}\text { \% Pres. } \\
2014\end{array}$ & $\begin{array}{c}\text { \% Pres. } \\
2018\end{array}$ \\
\hline $\begin{array}{l}\text { Población total } \\
\text { (proy. 2018) }\end{array}$ & $\begin{array}{l}-1,06 \mathrm{e}-07 * \\
(5,73 \mathrm{e}-08)\end{array}$ & $\begin{array}{l}-7,36 \mathrm{e}-08 \\
(5,10 \mathrm{e}-08)\end{array}$ & $\begin{array}{c}-2,38 \mathrm{e}-08 \\
(4,00 \mathrm{e}-08)\end{array}$ & $\begin{array}{l}-5,75 e-08 \\
(5,53 e-08)\end{array}$ & $\begin{array}{l}-1,67 \mathrm{e}-07 * \\
(8,94 \mathrm{e}-08)\end{array}$ & $\begin{array}{c}-2,12 \mathrm{e}- \\
07 * * \\
(9,17 \mathrm{e}-08)\end{array}$ \\
\hline $\begin{array}{l}\text { \% población rural } \\
\text { (proy.2018) }\end{array}$ & $\begin{array}{l}0,00786 \\
(0,0167)\end{array}$ & $\begin{array}{c}0,0361 * * \\
(0,0148)\end{array}$ & $\begin{array}{l}-0,00102 \\
(0,0114)\end{array}$ & $\begin{array}{l}0,0303 * \\
(0,0159)\end{array}$ & $\begin{array}{c}0,0704 * * * \\
(0,0260)\end{array}$ & $\begin{array}{c}0,0790 * * * \\
(0,0267)\end{array}$ \\
\hline $\begin{array}{l}\text { Capital de } \\
\text { departamento }\end{array}$ & $\begin{array}{c}0,0433 \\
(0,0311)\end{array}$ & $\begin{array}{c}0,0332 \\
(0,0276)\end{array}$ & $\begin{array}{c}0,0179 \\
(0,0235)\end{array}$ & $\begin{array}{c}0,0483 \\
(0,0304)\end{array}$ & $\begin{array}{l}0,0893 * \\
(0,0484)\end{array}$ & $\begin{array}{c}0,0781 \\
(0,0496)\end{array}$ \\
\hline Constante & $\begin{array}{l}0,414 * * * \\
(0,0581)\end{array}$ & $\begin{array}{l}0,421 * * * \\
(0,0517)\end{array}$ & $\begin{array}{l}0,300 * * * \\
(0,0442)\end{array}$ & $\begin{array}{l}0,509 * * * \\
(0,0589)\end{array}$ & $\begin{array}{l}0,838 * * * \\
(0,0906)\end{array}$ & $\begin{array}{l}1,220 * * * \\
(0,0929)\end{array}$ \\
\hline Observaciones & 905 & 903 & 823 & 882 & 905 & 904 \\
\hline $\mathrm{R}^{2}$ ajustado & 0,206 & 0,195 & 0,185 & 0,178 & 0,164 & 0,254 \\
\hline
\end{tabular}

Errores estándar entre paréntesis $* * * p<0.01, * * p<0.05, * p<0.1$

La tabla evidencia que, al controlar por los demás factores, en todas las elecciones el CD obtuvo una proporción significativamente mayor de los votos en los municipios con tradición conservadora. Dado que se trata de comicios en los que compiten una relativamente menor cantidad de opciones, es natural que en las dos elecciones presidenciales el efecto sea mayor. Los modelos muestran que, en promedio, el CD obtuvo cerca de 9 puntos porcentuales más en los municipios conservadores que en los demás en la primera vuelta presidencial de 2014, así como alrededor de 8 puntos porcentuales en la primera vuelta de 2018. No obstante, en elecciones con mayor número de competidores - como las de Congreso--, el efecto de la herencia conservadora también es notorio, entre poco menos de 3 puntos porcentuales (Cámara 2018) y casi 6 puntos (Senado 2014).

Estos resultados sugieren que, más allá de los factores asociados al conflicto armado y a las condiciones de desarrollo y pobreza del municipio, el uribismo ha echado raíces en municipios donde el PC se había hecho fuerte durante gran parte del siglo veinte. ${ }^{14}$

Pasando al análisis de los factores propuestos por las dos hipótesis alternativas, nuestros resultados sugieren que la relación entre la exposición a la violencia

14 En otro artículo en curso encontramos que el actual Partido Conservador sigue usufructuando sus reductos del pasado, compartiendo con el CD el dominio sobre esos municipios (Rodríguez-Raga y Wills-Otero, sf.). 
| La renovación del conservadurismo tradicional a través de nuevos partidos.

política y el respaldo electoral al CD, planteada en la hipótesis $\mathrm{H} 2$, es clara en el caso de las elecciones presidenciales; controlando los demás factores, este partido recibió en promedio casi siete puntos porcentuales menos en estos municipios en la primera vuelta presidencial de 2018 (tabla 7). En las elecciones de Congreso, aunque los coeficientes tienen el signo correcto, no alcanzan el nivel mínimo de significancia estadística.

En cuanto a la hipótesis H3, los resultados dan cuenta de un mejor desempeño electoral del Centro Democrático en municipios comparativamente con mayor desarrollo — medido por su PBI per cápita_-; los coeficientes son estadísticamente significativos en todas las elecciones. Esta relación se ve corroborada cuando se examinan los coeficientes de los modelos para la variable que mide la pobreza multidimensional de los municipios (tabla 7). Aunque estos no llegan al nivel mínimo de significancia en las elecciones presidenciales, en las de Congreso los resultados sí sugieren que, ceteris paribus, el cD obtiene menor respaldo electoral en los municipios más pobres.

Vale la pena subrayar que, a pesar de esta relación negativa entre pobreza y éxito electoral del $\mathrm{CD}$, los resultados de la tabla 7 también indican que este partido obtiene mayores réditos en los municipios menos desiguales, a juzgar por el signo negativo de los coeficientes correspondientes a las dos variables del indicador Gini, que alcanzan significancia estadística en varias de las elecciones analizadas. Los modelos sugieren que diez puntos más en el índice Gini municipal, por ejemplo, le significaron al CD casi 16 puntos porcentuales menos en la primera vuelta presidencial de 2018. Esta relación entre desigualdad contextual y respaldo electoral para el Centro Democrático, así como para otros partidos, sin duda merece una exploración más profunda de la que podemos ofrecer en este artículo.

\section{Conclusiones}

En este trabajo respondimos la pregunta sobre cuáles son los factores determinantes del caudal electoral del Centro Democrático en las elecciones nacionales en las que ha participado desde su creación en 2013. Además de probar hipótesis que otros trabajos han incluido en sus análisis, mostramos la plausibilidad de nuestro argumento enfocado en la estrecha relación que existe entre dicho caudal electoral y la tradición política asociada al PC. La conclusión es simple: el CD obtiene significativamente mejores resultados electorales en municipios que durante más de cinco décadas controló el Partido Conservador. La explicación que ofrecemos es que la clara agenda programática conservadora y de derecha del CD ha logrado atraer a los votantes en dichos municipios. Esto puede resultar del hecho de que estos votantes se han visto desencantados con un PC desideologi- 
zado, clientelizado y deslegitimado. El CD surgió como una alternativa partidista que, en sus pocos años de existencia, ha mostrado coherencia entre las propuestas que hace y las políticas que ejecuta. Así, votantes provenientes de municipos tradicionalmente conservadores, que tendrían preferencias ideológicas cercanas a los pilares que defiende el nuevo partido y su líder, Álvaro Uribe Vélez, habrían decidido apoyarlo electoralmente. Esta interpretación que hace referencia a la permanencia geográfico-temporal de una cierta cultura política en Colombia merece indudablemente una consideración y análisis con una profundidad mayor a la que podemos ofrecer en este artículo.

Futuras investigaciones podrían explorar también en qué medida, como resultado de la combinación de las identidades ideológicas mencionada antes y de cálculos estratégico-electorales, los cuadros políticos del CD actual en las regiones eran miembros del PC o provienen de familias o tradiciones conservadoras locales.

Este trabajo es un aporte significativo para entender el fenómeno del uribismo en Colombia y para avanzar en la comprensión de las características y de los factores coadyuvantes del surgimiento y consolidación de partidos personalistas de derecha en América Latina. La dimensión electoral que acá analizamos complementa estudios previos sobre los orígenes de este partido en los que el liderazgo del expresidente Uribe fue fundamental. Por otro lado, este trabajo desafía estudios académicos previos del ámbito colombiano que han concluido que después del Frente Nacional el país superó la división sectaria que por cerca de un siglo ocasionaron los partidos tradicionales. Según varios analistas, este pacto de élites produjo, entre otras cosas, la desideologización y clientelización de estos partidos. Aunque existe amplia evidencia que respalda esta visión, los hallazgos de este artículo sugieren otra hipótesis, quizás complementaria: si bien es posible que el clivaje por identidad partidista — que caracterizó al país durante la primera mitad del siglo veinte - sea cosa del pasado, quizás las concepciones fundamentales que tienen las personas sobre cómo debe funcionar el mundo, eso que podríamos llamar la ideología, ha permanecido y se ha reproducido; $\mathrm{y}$, aunque durante mucho tiempo fueron perdiendo su identificación con el PC, el CD ha llegado para tomar estas concepciones en adopción, reinterpretarlas y resignificarlas.

En esa medida. los resultados de este estudio pueden ser útiles para analizar otros partidos conservadores de América Latina que sufrieron declives electorales en las últimas décadas. Algunas preguntas que se pueden responder son las siguientes: ¿hasta qué punto, los votantes desencantados migraron hacia otras organizaciones partidarias que responden más claramente a sus demandas o interpretan de mejor manera sus preferencias? ¿Hasta qué punto, los partidos que 
$100 \mid$ La renovación del conservadurismo tradicional a través de nuevos partidos.

sufrieron declives electorales, hicieron transformaciones para adaptarse a las nuevas circunstancias? En caso dado, ¿qué tan exitosos han sido en la recuperación de su electorado? De manera más general, los resultados de esta investigación invitan a profundizar en los estudios sobre identidades y valores políticos y su reproducción en el tiempo. 


\section{Referencias}

Anria, S. (2019). When Movementes Become Parties. The Bolivian mas in Comparative Perspective. Cambridge, Reino Unido: Cambridge University Press.

Archer, R. (1995). Party Strength and Weaknesses in Colombia's Besieged Democracy. En Scott Mainwaring y Timothy Scully (eds.), Building Democratic Institutions. Party Systems in Latin America (164-199). Stanford, EE.UU.: Stanford University Press

Atehortúa, J. M. C. (2016). ¿Ésta es la paz de Santos?: el partido Centro Democrático y su construcción de significados alrededor de las negociaciones de paz. Revista CS, 19, 15-37.

Cifuentes, C. F., J. F. Pino. 2018. Conmigo o contra mí: análisis de la concordancia y las estrategias temáticas del Centro Democrático en Twitter. Palabra Clave, 21(3), 885-916.

Congreso Visible (2020): https://congresovisible.uniandes.edu.co/

Cyr, J. (2016). Between Adaptation and Breakdown. Conceptualizing Party Survival. Comparative Politics, 49(1), 125-145.

GamboA, L. (2019). El reajuste de la derecha colombiana. El éxito electoral del uribismo. Colombia Internacional, 99, 187-214.

García-SÁnchez, M., J. C. Rodríguez-Raga. (2019). Personality and an Internal Enemy: Understanding the Popularity of Álvaro Uribe, 2002-2010. Revista Latinoamericana de Opinión Pública, 8(2), 89-123.

Gibson, E. (1996). Class and Conservative Parties: Argentina in Comparative Perspective. Baltimore, EE. Uu.: Johns Hopkins University Press.

GonzÁlez, F. (1997). Aproximación a la configuración política en Colombia. Para leer la política. Ensayos de historia política colombiana. Tomo I. Bogotá, Colombia: cINEP.

Hartlyn, J. (1993). La Política del Régimen de Coalición. La experiencia del Frente Nacional en Colombia. Bogotá, Colombia: Tercer Mundo Editores y Ediciones Uniandes, CEI.

Levitsky, S., L. WAy. (2012). Beyond Patronage: Violent Struggle, Ruling Party Cohesion, and Authoritarian Durability. Perspectives on Politics, 10(4), 869-889.

Levitsky, S., J. Loxton, B. Van Dyck, Jorge Domínguez (Eds). 2016. Challenges of Party Building in Latin America. Cambridge; Reino Unido: Cambridge University Press. 
$102 \mid$ La renovación del conservadurismo tradicional a través de nuevos partidos.

Loxton, J. (2016). Authoritarian Successor Parties and the New Right in Latin America. En S. Levitsky, J. Loxton, B. Van Dyck, J. Domínguez (Eds.), Challenges of Party-Building in Latin America (245-272). Cambridge, Reino Unido: Cambridge University Press,

Loxton, J. (2021). Conservative Party-Building in Latin America. Authoritarian inheritance and counterrevolutionary struggle. Oxford, Reino Unido: Oxford University Press.

Losada, R., N. Liendo. (2016). El Partido Centro Democrático en Colombia: razones de su surgimiento y éxito. Análisis Político, 29(87), 49-51.

Luna, J. P. (2014). Segmented Representation: Political Party Strategies in Unequal Democracies. Oxford, Reino Unido: Oxford University Press.

Luna, J. P., C. Rovira Kaltwasser. (2014). The Right in Contemporary Latin America: A Framework for Analysis. En J. P. Luna y C. Rovira Kaltwasser (Eds.), The Resilience of the Latin American Right (1-22). Baltimore, EE. UU.: Johns Hopkins University Press,

Luna, J. P., R. Piñeiro Rodríguez, F. Rosenblatt, G. Vommaro. (2020). Political Parties, Diminished Sub-types and Democracy. Party Politics, 27(2), 294307.

Lupu, N. (2016). Building Party Brands in Argentina and Brazil. En S. Levitsky, J. Loxton, B. Van Dyck, J. Domínguez (Eds.). Challenges of Party Building in Latin America. Cambridge, Reino Unido: Cambridge University Press.

Mainwaring, S., A. M. Bejarano, E. Pizarro Leongómez (Eds.). (2008). La crisis de representación democrática en los países andinos: un panorama general. Bogotá, Colombia: Grupo Editorial Norma.

Melo, J. O. (2018). Historia Mínima de Colombia. Madrid, España: Turner Publicaciones.

Middlebrook, K. J. (2000). Conservative Parties, the Right, and Democracy in Latin America. Baltimore y Londres, EE. UU., Reino Unido: The Johns Hopkins University Press.

Milanese, J. P., A. Abadía, L. Manfredi. (2016). Elecciones regionales en Colombia 2015: análisis de las nuevas gobernaciones y asambleas departamentales. Revista Uruguaya de Ciencia Política, 25(1), 1-26.

Orjuela, L. J. (2015). Uribe versus Santos: del enfrentamiento personal a la diferenciación ideológica. Revista de Estudios Sociales, 52, 201-207. 
Ortiz-Ayala, A., M. García-SÁnchez. (2014). Porque te quiero te apoyo': Estilo de gobierno y aprobación presidencial en América Latina. Revista de Ciencia Política, 34(2), 373-398.

Pinzón de Lewin, P. (1989). Pueblos, regiones y partidos: 'la regionalización electoral': atlas electoral colombiano. Bogotá, Colombia: Uniandes, Cider, Cerec.

Roberts, K. M. (2012). Market Reform, Programmatic (De) alignment, and Party System Stability in Latin America. Comparative Political Studies, 46(11), $1422-1452$.

Rodríguez-Raga, J. C., L. Wills-Otero. (sf). Las raíces conservadoras del Centro Democrático en las elecciones nacionales y regionales, 2014-2019.

Rosenblatt, F. (2018). Party Vibrancy and Democracy in Latin America. Oxford, Reino Unido: Oxford University Press.

Samuels, D. (2006). Sources of Mass Partisanship in Brazil. Latin American Politics and Society 48(2), 1-27.

Samuels, D. y C. Zucco. (2014). The Power of Partisanship in Brazil: Evidence from Survey Experiments. American Journal of Political Science, 58(1), 212-225.

Vommaro, G. (2019). De la construcción partidaria al gobierno: PRo-Cambiemos y los límites del 'giro a la derecha' en Argentina. Colombia Internacional, 99(99), 91-120.

Vommaro, G. (2017). La larga marcha de Cambiemos. Buenos Aires: Siglo Xxi.

Vommaro, G., S. Morresi, A. Bellotti. (2015). Mundo Pro. Buenos Aires, Argentina: Planeta.

Weintraub, M., J. F. Vargas, T. E. Flores, T. E. (2015). Vote choice and legacies of violence: evidence from the 2014 Colombian presidential elections. Research \& Politics, 2(2). Doi: https://doi.org/10.1177\%2F2053168015573348.

Wills-Otero, L. (2014). The Right in Power in Colombia. The Government of Álvaro Uribe Vélez, 2002-2010. J. P. Luna, C. Róvira Kaltwasser (Eds.). The Latin American Right in the Neoliberal Age (194-218). Baltimore, EE. UU.:The Johns Hopkins University Press,

Wills-Otero, L. (2015). Latin American Traditional Parties, 1978-2006. Electoral Trajectories and Internal Party Politics, Bogotá, Colombia: Ediciones Uniandes. 
104 La renovación del conservadurismo tradicional a través de nuevos partidos.

WiLls-Otero, L. (2016). The electoral performance of Latin American traditional parties, 1978-2006: Does the internal structure matter? Party Politics, 2(6), 758-772. Doi: https://doi.org/10.1177\%2F1354068814563971

Wills-Otero, L. (2020). Party Systems in Latin America. Oxford Research Encyclopedia of Politics, 1-23.

Wills-Otero, L., B. Ortega, V. Sarmiento. (2021). The Colombian PL and PC: From Political Parties to Unrooted Parties. En J. P. Luna, R. Piñeiro, F. Rosenblatt y G. Vommaro. Diminished Parties. Democratic Representation in Contemporary Latin America (documento en proceso de publicación).

Los autores han realizado igual contribución al artículo y son los únicos responsables de su contenido. 